\title{
Resistance training and changes to plasma lipoproteins in postmenopausal women
}

\author{
Janet Erica Viljoen (MSc) \\ Candice Jo-Anne Christie (PhD) \\ Rhodes University, Grahamstown, South Africa
}

\begin{abstract}
Objectives. The main purpose of this study was to assess the effect of progressive resistance training on the blood lipid profile in postmenopausal women.

Methods. Twenty-six female participants aged $50-75$ years were selected from the population of Grahamstown, South Africa. All participants were previously sedentary and possessed at least one lipid profile abnormality but were otherwise healthy. Pretests included a sub-maximal stress test, stature, mass, central and limb girths as well as an oral glucose tolerance test (OGTT) and a total blood lipid profile. Participants took part in a 24-week progressive resistance training programme, consisting of three supervised sessions per week, each lasting 45 minutes. Participants were not permitted to lose more than $10 \%$ of initial body mass during the 24-week study. All pre-test measures, excluding the stress test and the OGTT, were repeated every 4 weeks for the duration of the study.

Results. Body mass, body mass index and waist-to-hip ratio did not change. Girth measures at mid-humerus, chest, waist, hip, mid-quadricep and mid-gastrocnemius all decreased significantly $(p<0.05)$. LDL-cholesterol increased significantly over the course of 24 weeks ( $3.61 \mathrm{mmol}^{-1} \mathrm{I}^{-1}$ to $4.07 \mathrm{mmol} . \mathrm{I}^{-1}$ ), as did total cholesterol $\left(5.81 \mathrm{mmol}^{-1} \mathrm{I}^{-1}-6.24 \mathrm{mmol} . \mathrm{I}^{-1}\right)$. Triglyceride concentration remained unchanged and HDL-cholesterol decreased significantly between the pre-test measure $\left(1.55 \mathrm{mmol}^{-\mathrm{I}^{-}} \mathrm{I}^{1}\right)$ and the measure after 6 months $\left.\left(1.42 \mathrm{mmol}^{-1}\right)^{-1}\right)$.

Conclusion. The blood lipid profile in a sample of postmenopausal women was not positively affected by a progressive resistance training programme over a 24 -week period.
\end{abstract}

\section{Introduction}

Morbidity and mortality related to sedentary living are increasing worldwide. ${ }^{1}$ In particular, coronary heart disease (CHD), which is closely linked to risk factors such as obesity, insulin resistance, high blood pressure and abnormal blood lipid profiles, is increasing.

Traditionally women have been thought to be exempt from CHD, especially pre-menopausal women. ${ }^{2}$ It is now understood that the risk of developing hypercholesterolaemia and $\mathrm{CHD}$ increases exponentially after menopause, and that it is vital for a woman's cholesterol levels to be closely monitored. ${ }^{3}$ Two modalities of treatment exist for hypercholesterolaemia: drug intervention and lifestyle intervention. As drug interventions can have unpleasant physical side-effects as well as economic implications and issues of availability, it is important to establish the effect of lifestyle interventions on improving the blood lipid profile in individuals at risk for CHD.

While the impact of diet on plasma lipoproteins is fairly well established, ${ }^{4}$ the effect of exercise is not as conclusive. ${ }^{5,6}$ While it appears that aerobic endurance training may be an effective means of managing hypercholesterolaemia, ${ }^{7-9}$ less is known about the effects of progressive resistance training. ${ }^{10,11}$ In response to resistance training, HDL-cholesterol was reduced in women aged $54-71$ years over 12 weeks. ${ }^{12}$ Further studies on samples including pre-menopausal women and men have either shown no change, ${ }^{8,14,15}$ positive change ${ }^{16}$ or, like Joseph et al., ${ }^{12}$ a negative response. There is very little literature has focused specifically on postmenopausal women. Thus the problem addressed in this research was to establish the effect of a 24-week progressive resistance training programme on the blood lipid profiles of a sample of previously sedentary postmenopausal women.

\section{Materials and methods Study design}

A prospective, longitudinal (24 weeks) study design was employed on a cohort of postmenopausal women. The participants were required to attend thrice-weekly sessions of supervised resistance training which progressed from $50 \%$ of the pre-test 1-repetition maximum (1RM) effort intensity to $80 \%$ of the $1 \mathrm{RM}$ intensity over the course of the 24-week period.

\section{Participant population}

Twenty-six participants were recruited via information disseminated through local newspapers as well as flyers at local pharmacies and at general practitioners' rooms. Volunteers all agreed to participate in a protocol approved by the Rhodes University, Grahamstown, South Africa Ethics Committee. Participants were females aged 50 - 75 years.

Inclusion criteria were: postmenopausal (cessation of menses at least 12 months prior to selection for the study), sedentary (defined 
as less than three regular sessions of physical activity of 30 minutes' duration per week for the previous 6 months, based on the American College of Sports Medicine's recommendation that three sessions of exercise weekly, each of 30 minutes, is minimally beneficial to the individual), free from heart, lung, liver and kidney disease, not on hormone replacement therapy and non-diabetic or pre-diabetic. The latter was assessed through an oral glucose tolerance test (OGTT) conducted prior to the study, results of which were used specifically to screen for irregularities and were not included as part of the study thereafter.

\section{Measurements}

Clinical evaluation included a stress echocardiogram (ECG), resting blood pressure while standing, sitting and supine, and a full physical examination by a medical practitioner. A full OGTT was carried out at a reputable pathology laboratory. Participants were required to fast from $22 \mathrm{~h} 00$ the evening before the OGTT, and presented at the laboratory at $08 \mathrm{~h} 30$. On arrival at the laboratory each participant was given a glucose solution to consume, mixed to specification (75 g of glucose mixed in water). A blood sample was taken within 2 minutes of consumption of the glucose drink and again 2 hours later. In between the two blood tests participants were permitted to leave the laboratory but were not permitted to eat or drink anything other than small amounts of water if required.

Anthropometric parameters included measures of stature, mass, and girth measures at the following anatomical sites: mid-humerus, chest over-bust, waist at the level of the umbilicus, hip, midquadricep and mid-gastrocnemius (Table I). Girth measures (upper arm, chest over-bust, waist, hip, thigh and calf) and mass (kg) were obtained by the principal researcher and were measured at the start of the same 60-minute exercise session on each occasion at 4-week intervals. Two measurements were taken and if these matched the researcher was satisfied that the measurement was valid. The following anthropometric indicators were calculated: waist-to-hip ratio and body mass index (Table I).

\section{TABLE I. Mean ( \pm standard deviation) demographic data obtained from the participants

$\begin{array}{llllll}\boldsymbol{N} & \begin{array}{l}\text { Age } \\ \text { (years) }\end{array} & \begin{array}{l}\text { Stature } \\ (\mathbf{m})\end{array} & \text { Mass }(\mathbf{k g}) & \begin{array}{l}\text { BMI } \\ \left(\mathbf{k g} \cdot \mathbf{m}^{-2}\right)\end{array} & \text { WHR } \\ 26 & 56.77 \pm 4.12 & 1.63 \pm 60 & 79.08 \pm 17.42 & 29.8 \pm 6.77 & 0.85 \pm 0.08\end{array}$

$\mathrm{BMI}=$ body mass index; WHR: waist-to-hip ratio.

Full, fasting blood lipid profiles were obtained at a reputable pathology laboratory. Blood was analysed using standard automated enzymatic processes on the Dimension Xpand Plus clinical chemistry system. The following serum determinations were made from samples collected in the morning after a 12-hour fast: total cholesterol, high-density cholesterol (HDL-C) and triglycerides (enzymatic method) and low-density lipoprotein cholesterol (LDL-C) $\left(\right.$ estimation ${ }^{16}$ ). For inclusion in the study, participants had to present with at least one lipid abnormality (total cholesterol higher than 5.20 mmol..$^{-1}$; LDL above $2.60 \mathrm{mmol}^{-1} \mathrm{I}^{-1}$ HDL above $1.30 \mathrm{mmol}^{-1} \mathrm{I}^{-1}$ or triglycerides above $1.70 \mathrm{mmol}^{-1} \mathrm{I}^{-1}$ ) and not present with an abnormal fasting blood glucose response. Measurements were repeated every 4 weeks.

\section{Training protocol}

A 24-week progressive resistance training (PRT) programme was designed engaging the 'FIND' principle (frequency, intensity, nature and duration). In order to ensure reliability and validity of results intensity was tightly monitored throughout the study. Initial intensity was set at $50 \%$ one-repetition-maximum (1-RM) and increased to $80 \%$ during the final phases of the project. The major muscle groups targeted are shown in Table II. Participants were also required to attend three sessions per week for the 24-week study trial. Although requested to maintain their habitual dietary intake for the duration of the study (and participants were regularly explained the reason as to why this was important), a limitation of the study is that diet was not monitored or controlled. This represents a weakness in the design of this study, of which the authors are aware. In executing this protocol the researcher intended to conduct a field study, replicating ordinary daily life so that the results might indicate the efficacy of lifestyle interventions in situ. In addition, this was a preliminary study for a further investigation, the design for which includes many improvements including better control of the participants' dietary intake.

\section{TABLE II. Exercises and musculature trained}

$\begin{array}{ll}\text { Exercise } & \text { Major muscles engaged } \\ \text { Lateral pull-down } & \begin{array}{l}\text { Latissimus dorsi, rhomboideus major, } \\ \text { trapezius }\end{array} \\ \text { Seated cable cow } & \begin{array}{l}\text { latissimus dorsi, trapezius, deltoid } \\ \text { group, erector spinae, external oblique, } \\ \text { rectus abdominus }\end{array} \\ \text { Leg extension } & \begin{array}{l}\text { Rectus femoris, vastus medialis, vastus } \\ \text { lateralis, vastus intermedius }\end{array} \\ \text { Chest press } & \begin{array}{l}\text { Pectoralis major, deltoid group, external } \\ \text { oblique, rectus abdominus, biceps } \\ \text { brachii, triceps brachii }\end{array} \\ \text { Abdominal crunches } & \begin{array}{l}\text { Rectus abdominus, external oblique, } \\ \text { internal oblique, transverse abdominus, } \\ \text { iliopsoas, multifidus (activation of the } \\ \text { core) }\end{array} \\ \text { Hamstring curls } & \begin{array}{l}\text { Semitendinosus, semimembranosus, } \\ \text { biceps femoris }\end{array} \\ \text { Step-ups } & \begin{array}{l}\text { Rectus femoris, vastus medialis, vastus } \\ \text { lateralis, vastus intermedius, semiten- } \\ \text { dinosus, semimembranosus, biceps } \\ \text { femoris, gastrocnemius, tibialis anterior }\end{array} \\ & \end{array}$

The exercise programme was divided into six phases of 4 weeks' duration (Table III). Each phase represented an increase in intensity, initially by increasing the intensity from $50 \%$ of the 1 -RM to $60 \%$ of the 1-RM, and then by increasing both the intensity and also the volume of repetitions and sets completed.

TABLE III. Exercise programme design

\begin{tabular}{lllllll}
\hline & \multicolumn{7}{c}{ Phase } \\
\cline { 2 - 7 } & $\mathbf{1}$ & $\mathbf{2}$ & $\mathbf{3}$ & $\mathbf{4}$ & $\mathbf{5}$ & $\mathbf{6}$ \\
Intensity (\%) & 50 & 60 & 60 & 70 & 70 & 80 \\
Sets (No.) & 1 & 1 & 2 & 2 & 3 & 3 \\
Repetitions (No.) & 12 & 12 & 12 & 12 & 12 & 12 \\
& & & & & & \\
\hline
\end{tabular}


Exercise sessions were offered at times self-selected by the participants. Each session was supervised by a research assistant (postgraduate students in the Department of Human Kinetics and Ergonomics, Rhodes University, Grahamstown). Participants were required to attend three sessions weekly, each lasting 45 - 60 minutes. The aerobic warm-up comprised $10 \%$ of the session time, and the PRT portion of the workout made up $90 \%$ of the session time. Inclusion in data analyses was dependent on the participant completing $80 \%$ of the sessions (72 sessions in total).

\section{Statistical analyses}

Data are presented as mean \pm standard deviation (SD). Statistical significance was set at $p \leq 0.05$. A repeated measures one-way analysis of variance (ANOVA) was employed to analyse the results, and statistical procedures were performed in Statistica $8 .{ }^{18}$

\section{Results}

\section{Compliance}

In order to be included in the data set participants had to attend at least $80 \%$ of the total number of sessions (72). Of the initial sample $(N=34) 87 \%$ completed $80 \%$ of the sessions and of this number, $9 \%$ attended $100 \%$ of the sessions. Of the remaining participants $3 \%$ attended $70-75 \%$ of sessions, and $10 \%$ had only attended $50-60 \%$ of the 72 sessions. Data from 26 participants were analysed once results had been corrected for compliance.

\section{Anthropometric and girth measures}

Body mass $(\mathrm{kg})$ did not display significant changes $(-0.77 \pm 0.62 \mathrm{~kg})$ over the course of 24 weeks $(p<0.05)$.

Waist circumference decreased significantly $(p<0.05)$ from baseline to 4 weeks $(-22 \pm 8 \mathrm{~mm})$ (Fig. 1). This measure, reflecting abdominal visceral fat (AVF) deposits, continued to decrease significantly at $8(-38 \pm 11 \mathrm{~mm}), 12(-48 \pm 19 \mathrm{~mm}), 16(-48 \pm 14 \mathrm{~mm})$, $20(-42 \pm 18 \mathrm{~mm})$ and 24 weeks $(-45 \pm 17 \mathrm{~mm})$. Hip girth reflected a similar pattern, also displaying significant decreases from week 8 $(-36 \pm 9 \mathrm{~mm})(p<0.05)$. Weeks $12(-35 \pm 9 \mathrm{~mm}), 16(-39 \pm 9 \mathrm{~mm}), 20$ $(-40 \pm 9 \mathrm{~mm})$ and $24(-41 \pm 8 \mathrm{~mm})$ were all significantly lower than the pre-test measure.

Upper extremity circumference showed significant decreases from week $8(-10 \pm 3 \mathrm{~mm})$ (Fig. 1). This reduction continued at weeks $12(-11 \pm 4 \mathrm{~mm}), 16(-12 \pm 3 \mathrm{~mm}), 20(-14 \pm 6 \mathrm{~mm})$ and $24(-14 \pm 6 \mathrm{~mm})$. Over-bust chest girth measure also showed a significant $(p=0.05)$ decrease at week $8(-23 \pm 14 \mathrm{~mm})$ and this trend continued through

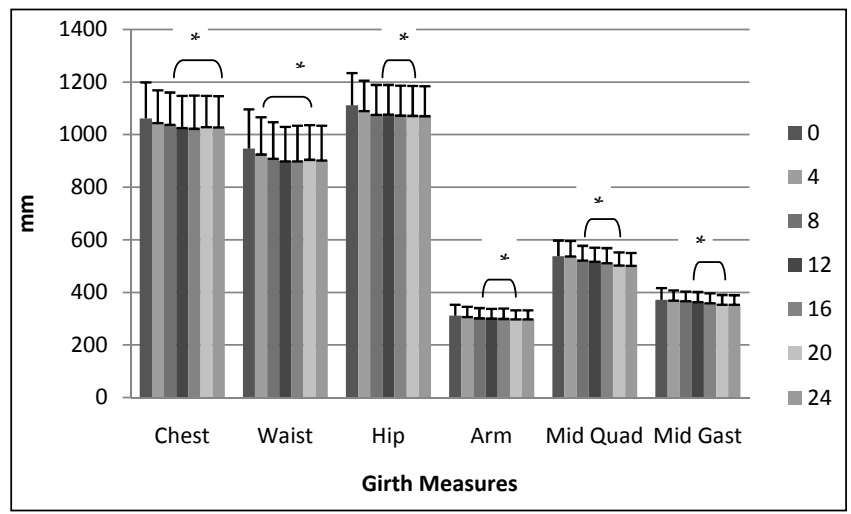

Fig. 1. Mean girth measures.

*Significant difference to baseline measure

Multiple values significantly different to baseline measures

Quad = quadriceps muscle group

Gast $=$ the gastrocnemius muscle weeks $12(-35 \pm 15 \mathrm{~mm}), 16(-39 \pm 11 \mathrm{~mm}), 20(-32 \pm 18 \mathrm{~mm})$ and 24 $(-33 \pm 18 \mathrm{~mm})$.

Mid-quadricep baseline measure $(537 \pm 60 \mathrm{~mm})$ decreased significantly at week $12(-19 \pm 3 \mathrm{~mm})(p<0.05)$. Significant decreases were also recorded at weeks $16(-25 \pm 3 \mathrm{~mm}), 20(-33 \pm 11 \mathrm{~mm})$ and $24(-34 \pm 11 \mathrm{~mm})$ (Fig. 1). Mid-gastrocnemius girth was significantly lower than the baseline measure at week $16(-13 \pm 6 \mathrm{~mm}), 20(-18 \pm 8$ $\mathrm{mm})$ and $24(-18 \pm 8 \mathrm{~mm})(p=0.05)$.

\section{Plasma lipoproteins}

Plasma lipoproteins measured by conventional methods reacted unexpectedly to the resistance training protocol. LDL increased significantly from baseline $\left(3.61 \pm 0.78 \mathrm{mmol}^{-1} \mathrm{I}^{-1}\right)$ to $4.07 \pm 0.81 \mathrm{mmol}^{-1} \mathrm{I}^{-1}$ $(p<0.05)$ following the 24-week PRT. While this is statistically significant it does not represent clinical significance, as the increase does not represent a 'risk category' increase. HDL decreased significantly at week $16\left(-0.06 \pm 0.03 \mathrm{mmol}^{-1} \mathrm{I}^{-1}\right)$ and continued to decrease at weeks $20\left(-0.07 \pm 0.06 \mathrm{mmol}^{-1} \mathrm{I}^{-1}\right)$ and $24\left(-0.13 \pm 0.06 \mathrm{mmol}^{-1} \mathrm{I}^{-1}\right.$. Triglycerides increased significantly $(p<0.05)$ at week $8(0.31 \pm 0.32$ mmol. $\left.I^{-1}\right)$ but thereafter decreased in the next 12 weeks, returning to pre-intervention values at conclusion of the study (Fig. 2). Total cholesterol increased steadily in the first 12 weeks, reaching significantly higher than baseline values at weeks $16\left(0.41 \pm 0.32 \mathrm{mmol}^{-1} \mathrm{I}^{-1}\right), 20$ $\left.\left(0.44 \pm 0.12 \mathrm{mmol}^{-1}\right)^{-1}\right)$ and $24\left(0.43 \pm 0.15 \mathrm{mmol}^{-1} \mathrm{I}^{-1}\right)$ (Fig. 2).

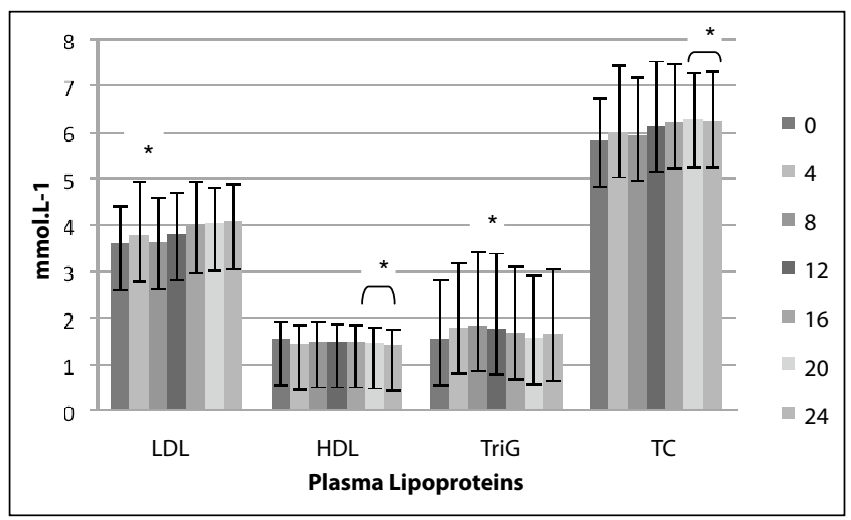

Fig. 2. Plasma lipoprotein changes over the course of 24 weeks.

*Significant difference to baseline measure

Multiple values significantly different to baseline measure.

\section{Discussion}

This study evaluated the effect of progressive resistance training on the plasma lipoproteins in postmenopausal women. Reliability of results required that the compliance of participants remained high. Thus, only those participants who achieved at least $80 \%$ attendance at exercise sessions were included in the data analyses. Furthermore, it was important for the outcomes of the study that the sample did not lose more than $10 \%$ of baseline body mass, as it has been demonstrated that weight loss positively influences plasma lipoprotein concentrations. ${ }^{19,20}$ Participants were instructed that loss of body mass greater than $10 \%$ would result in exclusion from the study and were requested not to attempt bodyweight loss. Body mass in the current cohort did not change over the duration of the experiment. Favourable changes in body composition can be expected as a result of resistance training, and in turn resting metabolic rate may increase. Subsequent loss of fat weight, but increase in muscle weight may have occurred, and may represent a benefit of resistance training in this cohort. Expectedly, BMI did not change significantly $\left(29.80 \pm 6.77 \mathrm{~kg} \cdot \mathrm{m}^{-2}\right.$ at baseline and $29.51 \pm 6.51 \mathrm{~kg} \cdot \mathrm{m}^{-2}$ 
after 24 weeks). Other girth measures were positively affected by the exercise programme. In particular, reductions at central and distal anatomical sites with a concomitantly stable body mass reflected a decrease in fat mass and a possible increase in fat-free mass (given that body mass remained stable). This is in contrast to the findings of Joseph et al., ${ }^{12}$ who found that 12 weeks of resistance training reflected no change in body composition in postmenopausal women, while in their age-matched male participants, body fat was reduced. At 12 weeks in the current sample, all girth measures were positively responding $(p<0.05)$ to the exercise programme.

Plasma LDL increased from as early as 4 weeks into the study (an increase of $0.17 \pm 0.36 \mathrm{mmol} . \mathrm{I}^{-1}$ compared with the pre-test baseline measure). This sharp increase leveled off at week $8(0.02 \pm 0.17$ mmol. $I^{-1}$ ) but by week $12 \mathrm{LDL}$ had increased by $6 \%$ relative to the baseline measure, and at week 24 LDL was significantly higher (12\%) than pre-test. The changes in LDL cholesterol were not supported by recent literature, ${ }^{12,21,22}$ which reported that resistance training in postmenopausal women resulted in no change to LDL levels. Important to note however is that the current study was 24 weeks in duration with this time frame resulting in a significant increase in LDL. At 12 weeks LDL had also not significantly altered effectively, suggesting that had the studies of Joseph et al,. ${ }^{12}$ Fahlmann et al. ${ }^{21}$ and Behall et al. ${ }^{22}$ been continued for longer, they too may have seen an increase in LDL levels. Furthermore, in the study by Joseph et al. body composition was not altered, unlike in this study where body composition positively changed.

HDL decreased significantly from baseline to 16 weeks $(-3 \%)$ and 24 weeks $(-8 \%)$, which is a finding supported by Joseph et al. ${ }^{12}$ In contrast to this it was found that resistance training had no effect on $\mathrm{HDL}$ concentrations in samples of middle-aged men. ${ }^{23}$

Triglyceride (TG) concentration reached a significant $20 \%$ $(p<0.05)$ above the baseline value at week $8\left(0.31 \pm 0.32 \mathrm{mmol}^{-1}\right.$ higher than the pre-test plasma triglyceride levels). Over the following 16 weeks the plasma concentration of triglycerides appeared to decrease steadily, returning to pre-test levels at 24 weeks. Similarly, in another study, no changes were found in triglyceride levels after 12 weeks of resistance training. ${ }^{12}$

The drop and then subsequent increase in TG concentrations must be viewed within the context of the study design. TG levels are known to be influenced by many lifestyle-related factors such as dietary intake, alcohol consumption, smoking and menstrual status, to name a few. ${ }^{4}$ While smoking and menstrual status were controlled for in this study, no controls were instituted for alcohol intake, dietary or any other lifestyle habits. The drop in TG after 8 weeks therefore could have been a consequence of subconscious (or possibly even conscious) altered habitual lifestyle as it is well known that when individuals start exercising, diet is often changed subconsciously and individuals tend to start living a healthier lifestyle. ${ }^{6}$ Motivation for healthier lifestyle habits may have reduced over time and hence the increase in TG levels back to baseline may, again, be due to poorer lifestyle choices outside of the exercise influence. Interestingly however is the fact that although body fat was not measured, it was postulated that there was a decrease in fat mass and an increase in LBM as reflected by the changes in girth measures. Evidence suggests that this should positively alter TG concentration ${ }^{13}$ and yet this was not the case. Either body composition was not altered due to the exercise intervention or the other lifestyle choices made by the participants could have negated the positive body composition effect. The latter is the more plausible.

Total cholesterol (TC) increased significantly $(p<0.05)$ by week 16 (an increase of $7 \%$ ) and was significantly elevated from baseline at both 20 and 24 weeks, which is in contrast to previous findings. ${ }^{14}$ Despite this significant change, it is important to note the clinical significance of these findings as at 24 weeks, the increase from baseline was only $0.43 \pm 0.15 \mathrm{mmol}^{-1} \mathrm{I}^{-1}$. Current recommended levels of the plasma lipoproteins are $\mathrm{HDL}>0.90 \mathrm{mmol}^{-1}{ }^{-1}, \mathrm{LDL} \leq 3.00 \mathrm{mmol} . \mathrm{I}^{-1}$ (but $\leq 1.50 \mathrm{mmol}^{-1} \mathrm{I}^{-1}$ for those with known cardiac risk), triglycerides $<1.70 \mathrm{mmol} . \mathrm{I}^{-1}$ and TC $\leq 5.00 \mathrm{mmol}^{-1} \mathrm{I}^{-1}$. The current sample fell within the 'borderline' category prior to the exercise intervention for LDL (3.61 mmol..$\left.^{-1}\right)$ and TC (5.81 mmol. $\left.\mathrm{I}^{-1}\right)$, and within the desirable range for both $\mathrm{HDL}(1.55 \mathrm{mmol} . \mathrm{l}-1)$ and triglyceride concentration $\left(1.54 \mathrm{mmol}^{-1} \mathrm{I}^{-1}\right)$. Following the training intervention these values had shown statistically significant changes $(p<0.05)$, but did not show clinically significant permutations. LDL remained within the 'borderline' category (4.07 mmol..$^{-1}$ at 24 weeks), HDL remained at desirable levels $\left(1.42 \mathrm{mmol}^{-I^{-1}}\right)$ and TC was still below $7.50 \mathrm{mmol}^{-\mathrm{I}^{-1}}$, which is the upper limit of the 'borderline risk' category $\left(6.24 \mathrm{mmol} . .^{-1}\right.$ at 24 weeks). TG concentrations above $2.26 \mathrm{mmol}^{-1} \mathrm{I}^{-1}$ would reflect hypertriglyceridaemia, ${ }^{27}$ but the TG concentrations for the current sample remained below this margin $\left(1.54 \mathrm{mmol}^{-1-}{ }^{1}\right.$ prior to the intervention and $1.65 \mathrm{mmol}^{-1} \mathrm{I}^{-1}$ at 24 weeks).

\section{Conclusion}

Although these findings suggest a negative lipoprotein response to resistance training in postmenopausal women, the findings should be interpreted within the context of the study design and in the light of clinical significance. This especially in light of the fact that dietary intake was not strictly controlled or monitored and that there was no comparative control group. Future studies should consider these factors. The large variation in results obtained indicates that a mechanism for positive change exists and requires a more strictly controlled study to become clearly evident.

\section{Acknowledgements}

The authors would like to acknowledge the contributions of Dr Celia P Jameson, Specialist Physician, for the health screening of the participants and Professor Sarah Radloff, Department of Mathematical Statistics, Rhodes University, for assistance with the statistical procedures. The authors would further like to thank the 2008 postgraduate students from the Department of Human Kinetics and Ergonomics at Rhodes University who assisted with supervision of the exercise sessions.

\section{Competing interests}

All authors declare that the answer to the questions on your competing interest form are all No and therefore have nothing to declare.

\section{Funding}

Funding for this research project was provided by the Joint Research Council at Rhodes University.

\section{REFERENCES}

1. Chakravarthy MV, Booth FW. Eating, exercise and the "thrifty" genotypes: connecting the dots toward an evolutionary understanding of modern chronic disease. J Appl Physiol 2000;96:3-10.

2. Skouby SO. Health in the menopause: advances in management. Intern Congr Ser 2004;1266:151-155.

3. Jensen J. Lipids and lipoprotein profile in postmenopausal women. Dan Med Bull 1992; 39:64-80.

4. Haskell WL. Cardiovascular disease prevention and lifestyle interventions: effectiveness and efficacy. J Cardiovasc Nurs 2003;18:245-255.

5. Halverstadt A, Phares DA, Wilund KR, Goldberg AP, Hagberg JM. Endurance exercise training raises high density lipoprotein cholesterol and lowers small low density lipoprotein and very-low density lipoprotein independent of body fat phenotypes in older men and women. Metabolism 2007;56:444-450. 
6. Trejo-Gutierrez $\mathrm{J}$ and Fletcher $\mathrm{G}$. Impact of exercise on blood lipids and lipoproteins. J Clin Lipidol 2007;1:175-181.

7. King AC, Haskell WL, Young DR, Oka RK, Stefanick ML. Long-term effects of varying intensities and formats of physical activity on participation rates, fitness and lipoproteins in men and women aged 50 to 65 years. Circulation 1995;91:2596-2604.

8. Kokkinos PF, Holland JC, Pittaras AE, Narayan P, Dotson CO, Papademetriou V. Cardio-respiratory fitness and coronary heart disease risk factor association in women. J Am Coll Cardiol 1995;26:358-364.

9. Spate-Douglas T, Keyser RE. Exercise intensity: its effect on the high density lipoprotein profile. Arch Phys Med Rehab 1999;80:691-695.

10. Nicklas BJ, Katzel LI, Busby-Whitehead J, Goldberg AP. Increases in high-density lipoprotein cholesterol with endurance exercise training are blunted in obese compared with lean men. Metabolism 1997;46:556-561.

11. Thompson PD, Yugalevitch SM, Flynn MM, et al. Effect of prolonged exercise training without weight loss on high-density lipoprotein metabolism in overweight men. Metabolism 1997;46:217-223.

12. Joseph LJO, Davey SL, Evans WJ, Campbell WW. Differential effect of resistance training on the body composition and lipoprotein-lipid profile in older men and women. Metabolism 1999:48:1474-1480.

13. Comizio $R$, Pietrobelli $A$, Tan $X Y$, et al. Total body lipid and triglyceride response to energy deficit: relevance to body composition models. AJP Endo 1998;274(5):E860-E866.

14. Elliott $\mathrm{KJ}$, Sale $\mathrm{C}$, and Cable NT. Effects of resistance training and detraining on muscle strength and blood lipid profiles in postmenopausal women. Br J Sports Med 2002;36:340-344.

15. LeMura LM, von Duvillard SP, Andreacci J, Klebez JM, Chelland SA Russo J. Lipid and lipoprotein profiles, cardiovascular fitness, body composition and diet during and after resistance, aerobic and combination training in young women. Eur J Appl Physiol 2000;82:451-458.

16. Wallace MB, Mils BD, Browning CL. Effects of cross-training on markers of insulin resistance/hyperinsulinaemia. Med Sci Sport Exerc 1997;29:1170-1175.
17. Friedewald WT, Levy RI, Fredrickson DS. Estimation of the concentration of low-density lipoprotein cholesterol in plasma, without use of the preparative ultracentrifuge. Clin Chem 1972;18:499-502.

18. StatSoft, Inc. STATISTICA 2007. Version 8.1 (www.statsoft.com)

19. Martini C, Pallottini V, De Marinis E, et al. Omega-3 as well as caloric restriction prevent the age-related modifications of cholesterol metabolism. Mechanisms of Aging and Development 2008;129:722-727.

20. Volek JS, Fernandez ML, Fernman RD, Phinney SD. Dietary carbohydrate restriction induces a unique metabolic state positively affecting atherogenic dyslipidaemia, fatty acid partitioning, and metabolic syndrome. Prog Lipid Res 2008;47:307-318.

21. Fahlmann MM, Boardley D, Lambert CP, Flynn MG. Effects of endurance training and resistance training on plasma lipoprotein profiles in elderly women. Journals of Gerontology A: Biological Sciences and Medical Sciences 2002;57:B54-60.

22. Behall KM, Howe JC, Martel G. Comparison of resistive to aerobic exercise training on cardiovascular risk factors of sedentary, overweight, premenopausal and postmenopausal women. Nutr Res 2003;23:607-619.

23. Smutok MA, Reece L, Kokkinos PF, et al. Aerobic versus strength training for risk factor intervention in middle aged men at high risk for coronary heart disease. Metabolism 1993;42:177-184.

24. McArdle WD, Katch FI, Katch VL. Exercise Physiology: Energy, Nutrition and Human Performanc,. 4th ed. 1996. Baltimore: Williams and Wilkins.

25. Hodgetts V, Coppack SW, Frayn KN, Hockaday TDR. Factors controlling fat mobilization from human subcutaneous adipose tissue during exercise. Am Physiol Soc 1991;71:445-451.

26. Brooks GA, Mercier J. Balance of carbohydrate and lipid utilization during exercise: the "crossover" concept. Am Physiol Soc 1994;76:2253-2296.

27. Eberly LE, Stamler J, Neaton JD. Relation of triglyceride levels, fasting and non-fasting, to fatal and non-fatal cardiovascular heart disease. Arch Intern Med 2003;163:1077-1083. 